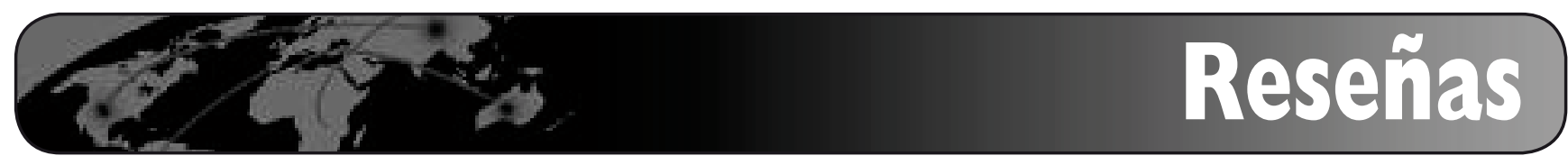

\title{
Encuentro de académicos, estudiantes y profesionales de la información en la Universidad de León
}

\author{
Por Blanca Rodríguez-Bravo
}

\begin{abstract}
Rodríguez-Bravo, Blanca. "Encuentro de académicos, estudiantes y profesionales de la información en la Universidad de León". El profesional de la información, 2009, enero-febrero, v. 18, n. 1, pp. 93-103.

DOI: 10.3145/epi.2009.ene13

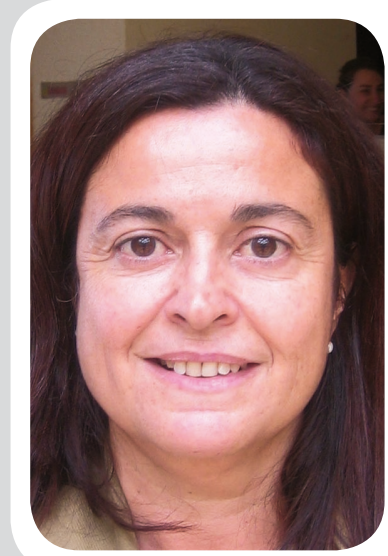

Blanca Rodríguez-Bravo, profesora titular de universidad del Área de Biblioteconomía y Documentación de la Universidad de León, fue la organizadora de este Encuentro.

Blanca Rodríguez imparte docencia en el campo de análisis y lenguajes documentales. Es autora de dos libros, coordinadora de otros dos y ha publicado cincuenta artículos, participaciones en monografías y comunicaciones de congresos referidas a la representación y organización de contenidos, evaluación de interfaces de opacs y plataformas-e, y oferta y consumo de recursos electrónicos de la comunidad académica.

Ha dirigido ocho proyectos subvencionados por el $M$ o de Educación español, la Junta de Castilla y León, y la Univ. de León, y ha participado en otros tres.
\end{abstract}

TUVO LUGAR EN la Facultad de Filosofía y Letras, Universidad de León, los días 17-18 de octubre de 2008.

$\mathrm{Su}$ objetivo era reflexionar sobre los requisitos que el mercado laboral exige de los graduados en Información y Documentación y determinar las necesidades previstas a corto-medio plazo, así como conocer las lagunas y/o deficiencias de los planes de estudio en vigor. Con las intervenciones de las dos mesas redondas quedó realizado un análisis muy completo de la profesión:

\section{Mesa 1. Principales competencias demandadas por el mercado del sector de la información}

Baiget comenzó refiriéndose al conocido problema del nombre, que cree que sigue siendo un gran lastre. La cantidad de denominaciones que se han ido inventando para re-

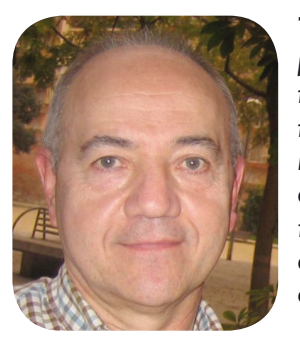

Tomàs Baiget, responsable de proyectos del Institut d'Estadística de Catalunya, creador y moderador de IweTel, fundador y director de EPI (El Profesional de la Información).

ferirse a las nuevas actividades que van asumiendo los profesionales de la información da una idea clara de lo mucho que ha evolucionado la profesión. A los bibliotecarios se les queda anticuado el nombre para poder ejercer los nuevos trabajos, muchos de los cuales ya no tienen nada que ver con la biblioteca. En este aspecto, otras carreras han tenido mejor suerte con los cambios tecnológicos: médicos, abogados, ingenieros..., pueden seguir usando su misma denominación como hace un siglo, sin confusión.

Nos encontramos pues ante un nombre ampliamente cuestionado, sobre todo desde que las tecnologías empezaron a cambiar los soportes y los canales de la información en los 70s.

Tantos nombres demuestran un gran polifacetismo de los "nuevos bibliotecarios" - de hecho, la carrera es un buen comodín para ejercer de cualquier cosa-, pero también muestran su crisis de identidad: "Vale, puedo hacer muchas cosas, pero ¿realmente qué soy?”.

"Tener una denominación de la profesión que depende de un lugar de trabajo concreto (biblioteca), ha sido un impedimento para evolucionar a otros entornos"

\section{Algunas denominaciones}

infomediario, infonomista, gestor de información, gestor de contenidos, gestor de conocimiento, experto o especialista en información, arquitecto de información, científico de la información, analista de información, gatekeeper, bibliotecnólogo, cibertecario, entrenauta..., sin olvidar las combinaciones de diversos nombres para especialidades como inteligencia competitiva, vigilancia tecnológica. 
El mundo de la ByD es el que está sufriendo un cambio más drástico. Todas las profesiones tienen que actualizarse con las nuevas herramientas y métodos, pero la gente de ByD además tiene que volver a inventar la profesión en sí. La sociedad de la información nos afecta mucho más. El profesionalista Andrew Abbott (2000) dice que hay tres contextos que afectan y determinan las profesiones:

1. Fuerzas socio-culturales y técnicas, que son las que crean la necesidad de una profesión. En ByD hemos visto cómo las personas aprenden a buscar su propia información (bricolage documental dentro del ámbito más general de amateurización de la masa), y tienen menor necesidad de la mediación de expertos. Los actuales usuarios de nuestras bibliotecas están mejor formados, lo que obliga (o permite) a los bibliotecarios llevar a cabo otras tareas.

2. La competencia de otras profesiones. En ByD en especial de los informáticos.

3. Habilidades (máquinas y organizaciones) que asumen el trabajo que antes hacían individos.

Dentro del punto 1 anterior habría que destacar el movimiento general de "desintermediación", que ha sido una tendencia extendida en el mercado de la información desde la década de los 90: autores, editores, productores de bases de datos, hosts distribuidores, agregadores..., todos intentan saltarse la clásica cadena de la información y llegar a los clientes sin intermediarios.

\section{"Los estudios reglados de ByD, ambición, curiosidad, Office e inglés constituyen la fórmula mágica para encontrar un buen trabajo"}

Aunque las necesidades (y las nuevas oportunidades) son más visibles para la puestos de trabajo tecnificados, no hay que olvidar que en la profesión son necesarios diferentes perfiles, y los "antiguos" seguirán vigentes.

La imagen que los actuales profesionales proyectan hacia la sociedad, atrae a un tipo de estudiante que tiende a perpetuar la misma imagen. Esto no es negativo, pero sí que ralentiza que en la carrera aparezcan candidatos más idóneos para cubrir las nuevas oportunidades.

Para terminar Baiget recomendó a los estudiantes:

- más ambición y buen marketing personal;

- entusiasmo y curiosidad para descubrir y probar nuevos sistemas de información;

- conocer perfectamente la suite Office (Word, Excel, Access, tablas, gráficos, PowerPoint);

- saber bien inglés escrito y hablado.

Tomàs Baiget, Institut d'Estadística de Catalunya, Via Laietana 58, 08003 Barcelona

Tel.: +34-934 120 088; fax: 934 123145

baiget@sarenet.es

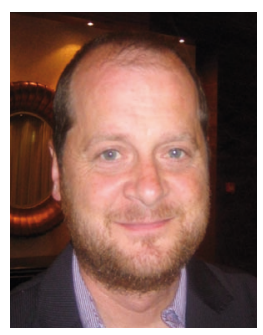

José-Antonio MerloVega, director del Servicio de Archivos y Bibliotecas de la Universidad de Salamanca. Vicepresidente de APEI (Asociación de Profesionales y Especialistas de la Información) y secretario de Fesabid.

Agradezco que la casa en la que aprendí el oficio de profesor me dé la oportunidad de reflexionar en voz alta sobre nuestra profesión, -empezó Merlo-. En muchas ocasiones se nos olvida que pertenecemos a uno de los colectivos más útiles para la ciudadanía; una profesión que ayuda a las personas, ofrecién- doles información seleccionada, procesada, cualificada.

Hace unos años, en un foro similar a éste, tuve la ocasión de hacer una radiografía de nuestro oficio desde la óptica de la España de hoy. No voy a entrar en su contenido, pero os invito a que consultéis $^{1}$ mi visión de lo que entonces califiqué y argumenté como una profesión delimitada, formada, informada, multidisciplinar, pequeña, institucional, inadvertida, desconocida, joven, cambiante, vocacionada y cooperativa. En esta ocasión prefiero aprovechar el ambiente de celebración de los primeros años de andadura de los estudios de Biblioteconomía y Documentación de la Universidad de León para volver sobre un decálogo de sugerencias que en aquel momento ofrecí sobre las cualidades de un buen profesional de la información y que hoy quiero volver a presentar "revisitado", dado que estamos ante un auditorio de estudiantes y jóvenes profesionales.

\section{Diez consejos para profesionales de la información}

Los profesionales de la información que realizan convenientemente su función, desde mi perspectiva, son los que cumplen de forma positiva estas condiciones:

1. Estar formados. La formación continua, el aprendizaje a lo largo de la vida, es una característica de la sociedad actual, en la que la evolución tecnológica y las posibilidades instrumentales avanzan rápidamente, especialmente en nuestros campos. El profesional de la información nunca termina su formación, debe actualizar su educación especializada constantemente.

2. Estar informados. Somos un colectivo y comunicarnos con nuestros colegas es una excepcional vía de intercambio de experiencias y conocimientos; por ese 
motivo es esencial leer revistas, boletines o blogs profesionales, así como participar en foros y estar suscritos a listas de distribución específicas.

3. Estar presentes. Trabajamos para organizaciones, por lo que debemos ser parte de las mismas, integrándonos en su órganos de decisión, comisiones o cualquier otro grupo en el que se puedan tomar decisiones o asesorar sobre políticas de información. Debemos llevar nuestra opinión allí donde se decida sobre nuestro trabajo.

4. Ser competentes. Hacer trabajo de forma correcta nos garantiza el respeto a nuestra actividad y el reconocimiento a los profesionales de la información. La calidad en el trabajo desempeñado es algo que debe exigirse a cualquier especialista y es algo, además, que debemos exigirnos nosotros mismos.

5. Ser activos. Las necesidades de información son constantes, pero la sociedad es cambiante, la tecnología evoluciona y las posibilidades de atención mejoran. El profesional de la información debe ser proactivo, adecuando los servicios a sus usuarios e inventando, creando o redefiniendo sistemas de información para nuestros ciudadanos y organizaciones.

6. Ser cooperantes. Los profesionales de la información están acostumbrados a trabajar en colaboración con otros miembros del colectivo. Así debe ser. Nuestros usuarios tienen necesidades muy similares, por lo que estar en constante contacto con centros parecidos, intercambiar prácticas e integrarse en redes redundará en la calidad de nuestra actividad.

7. Saber adaptarse. El cambio en nuestra profesión es constante y necesario. Debido sobre todo a las innovaciones tecnológicas, el profesional de la información debe aprender a reconvertir su trabajo, ya que las organizaciones se reestructuran y los medios evolucionan. Es habitual que haya que replantearse el sentido de nuestra actividad, por lo que hay que estar preparados para el cambio.

8. Saber aliarse. Los profesionales de la información trabajan para colectivos muy diversos a los que se debe apoyar y de los que se puede aprender. Será útil y enriquecedor disponer de sistemas de colaboración con personas u organizaciones de ámbitos diferentes a la documentación.

9. Saber difundirse. El reconocimiento no es posible sin un conocimiento previo. Estar presentes en los canales de información de nuestra entidad, así como en los medios informativos -profesionales o no- serán vías para dar a conocer nuestra actividad. Publicar una nota de prensa, aparecer en los medios locales, presentar una comunicación en un congreso o informar desde la web contribuirán a que se conozca nuestra labor.

10. Sentirse importantes. Una entidad está perdida sin un correcto sistema de gestión del conocimiento. Una sociedad está vacía sin un adecuado sistema de información pública. Los profesionales de la información deberían sentirse orgullosos de ejercer una profesión necesaria para el funcionamiento de las organizaciones, para la promoción de la cultura y para el desarrollo social y personal de los ciudadanos.

Son algunos consejos para que nuestro colectivo sea valorado, porque tenemos valor.

1. Merlo-Vega, José-Antonio. La profesión de archivos, bibliotecas y centros de documentación. En: La profesión de archivos, bibliotecas y centros de documentación. Jornada estratégica de revitalización. Bilbao: Aldee, 21 de septiembre de 2006.

http://eprints.rclis.org/7266

José-Antonio Merlo-Vega, Servicio de Archivos y Bibliotecas, Univ. de Salamanca, Pl. Univ. de Bolonia s/n, Fac. de Derecho, Planta Sótano, Campus Miguel de Unamuno, 37007 Salamanca.

Tel.: +34 923294 580; fax: 923 294582.

merlo@usal.es

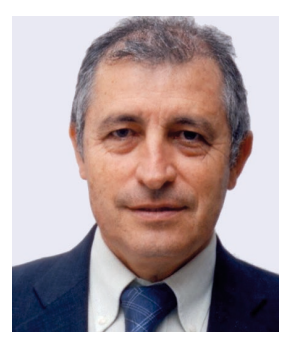

José-Antonio Moreiro-González, decano de la Facultad de Humanidades, Comunicación y Documentación de la Universidad Carlos III de Madrid, y vocal de Sedic.

Presentó un avance de resultados del estudio "Las competencias profesionales en Información y Documentación según las ofertas de empleo", que realizan José Antonio Moreiro, Juan Llorens, Jorge Morato, Sonia Sánchez y Valentín Moreno (Universidad Carlos III de Madrid) y Carlos Tejada (Universidad Complutense de Madrid).

El estudio analiza 362 ofertas de trabajo aparecidas en el Servicio de Orientación y Promoción Profesional (SOPP) de la UC3M, y de 1209 ofertas del blog Documentación (de Informa Catorze, dedicado al mundo laboral y formativo en el sector ByD), de los años 2005, 2006 y 2007.

http://www.documentacion.com.es/

El estudio se ha llevado a cabo dentro de las tareas del proyecto "Construcción de un sistema para la definición de perfiles profesionales y de competencias de formación para estudiantes de Información y Documentación: estudio comparativo para Brasil y España”, sub-

\section{"Estamos elaborando un tesauro de ofertas, a las que asociamos competencias y salarios"}


vencionado por el Ministerio de Educación dentro del Programa Hispano-Brasileño de Cooperación Interuniversitaria.

Se está realizando un tesauro jerarquizado de las ofertas, a las que se asocian las competencias y los salarios. Todas las ofertas estudiadas provienen de empresas (otra investigación diferente sería el análisis de las competencias requeridas por la oferta pública de empleo).

Los resultados que Moreiro avanzó son todavía de la primera fase descriptiva de las ofertas.

De las competencias cognitivas que se solicitan destacan dos por su rareza: analizar datos procedentes de estudios científicos y resolver problemas matemáticos.

En cuanto a aptitudes, más del $80 \%$ de las ofertas consideran necesarias:

- Capacidad de organización;

- Capacidad de involucrarse en el trabajo;

- Planificación del tiempo y de su trabajo;

- Ser metódico y sistemático en los procedimientos;

- Recoger e integrar información desde diversas fuentes (anuarios, revistas, internet); y

- Llevar a cabo tareas muy diferentes simultáneamente.

Entre las competencias instrumentales, la más presente es manejar internet para la búsqueda eficaz de información (86,3\%). Otras por encima de la mitad de las ofertas son:

- Conocer fuentes documentales y de bases de datos (tres de cada cuatro); y

- Utilizar programas informáticos específicos de su área.

El uso de otros idiomas fue mencionado en la mitad de las ofertas.

Como habilidades interpersonales aparecen señaladas con mayor frecuencia:

\section{En el $66 \%$ de las ofertas de trabajo de ByD se pide saber ofimática}

- Lealtad, honestidad y discreción;

- Facilidad para relacionarse con otras personas;

- Integración en un equipo de trabajo;

- Mantener la compostura en situaciones difíciles.

Respecto a las herramientas informáticas utilizadas, sorprende que las más generalizadas sean los procesadores de texto (dos tercios) y la ofimática en general, y que los programas informáticos concretos sólo fueron citados por la mitad de las ofertas. Además sólo algunos de ellos corresponden a sistemas de bases de datos, documentación, etc. Casi la mitad de las ofertas menciona otros programas específicos: Cumulus, Documentum, Unicorn, Libermarc, Oracle, SQL, Absys, Knosys, QuarkXPress...

Mientras que las tareas más demandadas se centran en las actividades de catalogación, atención a usuarios, búsqueda documental y mantenimiento de bases de datos.

José Antonio Moreiro González, Universidad Carlos III de Madrid.

Departamento de Biblioteconomía y Documentación.

C/Madrid, 126. 28903 Getafe, Madrid.

Tel.: +34-916 249 238; fax: 916 249212

joseantonio.moreiro@uc3m.es jamore@bib.uc3m.es

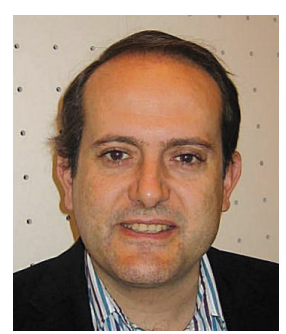

Luis Rodríguez-Yunta, Centro de Ciencias Humanas y Sociales, Unidad de Análisis Documental y Producción de Bases de Datos ISOC. Secretario general de Sedic.
En su intervención destacó las aportaciones realizadas desde Sedic al debate sobre las competencias, así como al conocimiento de las oportunidades de empleo:

- Participación en el proyecto europeo de sistematización de las competencias profesionales, en cuatro niveles, auxiliar, técnico, técnico superior y experto. Sedic publicó la versión española de las Eurocompetencias.

- Encuestas a los socios de Sedic (2001 y 2006), en las que se incluyen algunas preguntas sobre el acceso al empleo. Destaca la importancia de la experiencia como factor para la selección. En el medio empresarial, las relaciones interpersonales son el principal medio de acceso.

- Encuesta a empresas de servicios documentales (2004), en cuyos datos se percibe igualmente la importancia de la experiencia y las relaciones personales.

- Bolsa de trabajo de Sedic, en la que las demandas más frecuentes son perfiles de especialidad muy concreta, experiencia con programas informáticos y dominio experto del inglés.

\section{"Deberíamos contar con instituciones prestigiosas que puedan hacer una función de semillero formando buenos técnicos"}

"De la experiencia de trato con el medio empresarial podemos deducir que los empleadores buscan elementos que les permitan depositar su confianza en las personas seleccionadas. Por ello, debemos preguntarnos cómo podemos conseguir esta confianza, un aspecto en el que también influyen decisivamente las actitudes personales y 
la imagen colectiva" - dijo Rodríguez-Yunta. "Es difícil superar la imagen tópica de los bibliotecarios, y la dispersión de denominaciones profesionales en la empresa tampoco ayuda".

Como ideas para mejorar la imagen colectiva sugirió:

- Apostar por realizaciones concretas que poder mostrar, no sólo títulos.

- Realizar cursos específicos, no un conglomerado de asignaturas tratadas con superficialidad.

- Contar con empresas o instituciones que puedan hacer una función de semillero, de modo que puedan aportar un prestigio a todas las personas que pasen por ellas.

- Tener presencia en nuevos campos emergentes, en los que hay que competir con otros profesionales: web 2.0, web semántica, vigilancia tecnológica, bibliotecas digitales.

- Constituir empresas de servicios documentales que puedan afrontar la creciente externalización de funciones básicas en las organizaciones.

En definitiva, construir la imagen del futuro de la profesión sobre la base de la profesionalidad y la innovación.

Luis Rodríguez-Yunta, Centro de Ciencias Humanas y Sociales, Albasanz 26-28, 28037 Madrid.

Tel.: +34-916022 695

luis.ryunta@cchs.csic.es

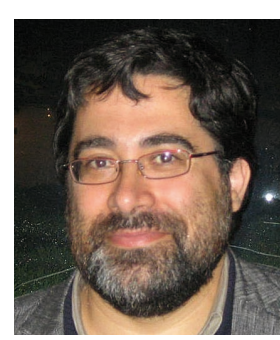

Cristóbal Urbano, decano de la Facultat de Biblioteconomia i Documentació de la Universitat de Barcelona.

Urbano considera que el análisis de las competencias demandadas por el sector de la información, no sólo es un compromiso de calidad sino también un imperativo legal para los nuevos títulos de Grado.

En el contexto de cambio de los planes de estudio en España para adaptarlos al Proceso de Bolonia ${ }^{1}$, la identificación de las competencias que ha de poseer un titulado de grado en Información y Documentación ya fue objeto de estudio en profundidad en el Libro Blanco Aneca $(2004)^{2}$ en el que participaron las 16 universidades españolas que en ese momento impartían o bien la Diplomatura en Biblioteconomía y Documentación, o bien la Licenciatura de segundo ciclo en Documentación. La actualización de dicho trabajo y la adaptación al entorno concreto de cada universidad se está llevando a cabo por los equipos y comisiones que actualmente desarrollan su proyecto concreto de titulación. Por ello, debates como el celebrado en León son de gran interés.

De todas formas, cabe destacar que se trata de una reflexión que se hace necesaria no solamente en razón del diseño de los nuevos títulos para adaptarlos al EEES (Espacio Europeo de Educación Superior) en el horizonte del 2010, tomado como el punto final de un proceso. Se ha de entender que la misma filosofía de dicho Espacio Europeo obliga a que discusiones como las realizadas en León se mantengan de forma continuada en el futuro, ya que la nueva legislación universitaria española contempla una fórmula más abierta y dinámica para la creación, modificación y actualización de títulos universitarios, lejos de la rigidez con la que hasta el momento se ha venido trabajando: recordemos que hasta la entrada en vigor de la reforma "Bolonia" en la que nos encontramos, los títulos tenían que acomodarse a unas directrices de titulaciones publicadas por el Ministerio en el $B O E$, que no se actualizaron nunca (así, las de la Di- plomatura datan de un Real Decreto de 1991 y las de la Licenciatura de uno de 1992). Es más, en el nuevo marco que se está construyendo, la acreditación externa de los resultados de las titulaciones constituye un elemento clave -inclusive, según la legislación, de supervivencia como título oficial en una determinada universidad-, que redunda en la idea misma del tema de la mesa redonda que nos ocupó el pasado 17 de octubre en León.

\section{"La filosofía del EEES obliga a que discusiones como las de León se mantengan continuadamente en el futuro"}

El nuevo modelo, que traslada una mayor responsabilidad y autonomía en el diseño de cada título a la universidad que lo propone, ha de redundar sin duda en el mantenimiento de la tensión intelectual entre el mundo académico y el mundo profesional: en el futuro, el proyecto formativo de una titulación universitaria española dependerá de la lectura dinámica y permanente del corpus académico de una disciplina a la luz de unos puntos de vista diversos (estudiantes, egresados, profesionales, empleadores, académicos, etc.) y en un marco más internacional. Por todo ello, en el futuro se tendrán que considerar como instrumentos imprescindibles el establecimiento de consejos asesores externos de las titulaciones o de las facultades (formados por empleadores, profesionales independientes, asociaciones profesionales y antiguos alumnos), los estudios de inserción profesional y de satisfacción de los titulados, así como encuentros similares al que estamos reseñando.

Esta nueva dinámica universitaria más abierta y flexible, si bien 
es común a todas las titulaciones que en el futuro existan en nuestro país, es especialmente relevante en el ámbito de la Información y Documentación: a nadie se le oculta que los cambios que el entorno digital en red introduce en nuestros perfiles profesionales y en nuestros objetos de estudio, son de un calado superior al de otras disciplinas, en las que los instrumentos de trabajo o de intervención evolucionan, pero en las que los objetos de estudio tienen una mayor estabilidad (por ejemplo, pensemos en las diferencias de afectación que internet ha comportado en nuestra área en comparación con la Odontología!). En definitiva, nos encontramos ante un nuevo escenario en el que el mundo académico ha de saber dialogar con el entorno profesional, especialmente en nuestro caso.

\section{Notas}

1. Véase el documento divulgativo del Ministerio: Marco, enfoque y calendario hacia el EEES [en línea]: Real Decreto 1393/2007 por el que se establece la ordenación de las enseñanzas universitarias oficiales. Madrid: Ministerio de Educación y Ciencia, 2008. http://web.micinn. es/files/2008-eees.pdf

2. Libro blanco título de Grado en Información y Documentación [en línea]. Madrid: Aneca, 2004. http://www.aneca.es/activin/docs/libroblanco_ jun05_documentacion.pdf

Cristóbal Urbano, Facultat de Biblioteconomia i Documentació, Univ. de Barcelona, Melcior de Palau 140, 08014 Barcelona.

Tel.: 934035 770; fax: 934035 772

urbano@ub.edu

\section{Mesa 2. Fortalezas y debilidades de la formación de los Diplomados en Biblioteconomía y Documentación y Licenciados en Documentación}

La Universidad de León invitó a un grupo de profesionales, antiguos alumnos de la Diplomatura en

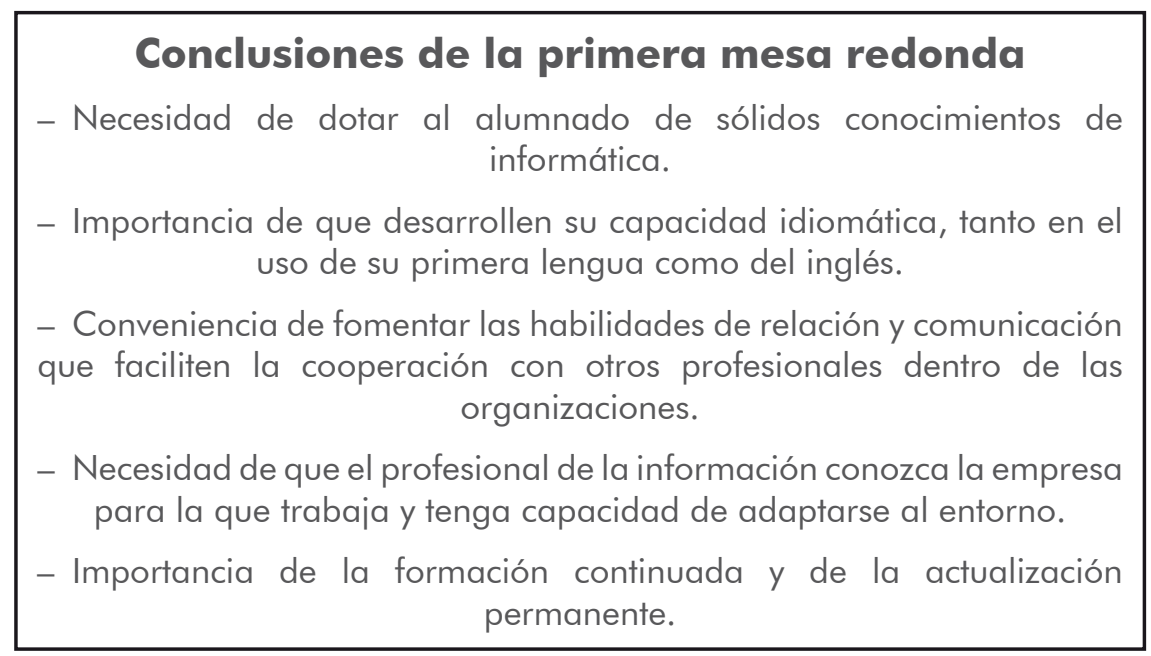

Biblioteconomía y Documentación, a que reflexionasen, tras varios años de experiencia profesional, sobre los aspectos positivos y negativos de la formación que habían recibido.

Se eligieron alumnos de las cinco primeras promociones, que finalizaron su diplomatura entre 1993 y 1997, porque los organizadores consideraron más conveniente que expusieran sus opiniones profesionales que ya tuvieran una trayectoria significativa. En la elección de las personas concretas se atendió a que estuviera representada la empresa privada y la pública al igual que los principales sectores: archivos, bibliotecas y centros de documentación especializados.

\section{Desde una biblioteca universitaria}

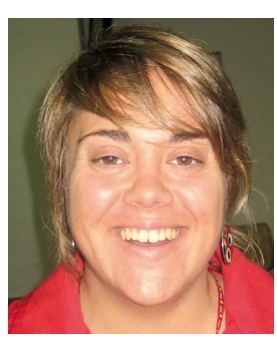

Leticia BarrionuevoAlmuzara, responsable de la Biblioteca de Filosofía y Letras, Área de Humanidades y Ciencias Sociales, Universidad de León

Cursó la Diplomatura durante el periodo 1994-1997, y considera que el Plan de Estudios entonces vigente representó una debilidad por la escasa especialización de las asignaturas, pero es muy optimista respecto los nuevos grados de Infor- mación y Documentación, los cuales "se convertirán en fortaleza".

Sin embargo, de cara a preparar oposiciones piensa que la formación que recibió durante los tres cursos de Diplomatura resultó bastante adecuada y le sirvió como cimiento de lo que luego sería un temario completo y ampliado, con la bibliografía correspondiente.

Destacó las falta de clases prácticas durante los tres cursos.

En cuanto a fortalezas se refirió a la asignatura de Catalogación, durante primero y segundo, y sobre todo las becas de formación y cooperación que la Universidad ofrecía en las distintas empresas e instituciones colaboradoras, como único contacto que el alumno tenía con el mundo profesional.

Lanzó varias preguntas al público, que quedaron sin respuesta: ¿Se trata de una profesión vocacional?, ¿cuál debe ser el enfoque: Biblioteca, Archivo, o Servicio de Información?, ¿mirando hacia la empresa pública o la privada?...

Concluyó la exposición con las cualidades que a su parecer debe tener un profesional de la información del siglo XXI:

Intuitivo, Persistente, Emprendedor, Proactivo, Diligente, Perfecto conocimiento de herramientas web y Saber venderse como profesional. 
Leticia Barrionuevo, Biblioteca, Facultad de Filosofía y Letras, Universidad de León. 24071 León.

Tel.: +34-987 291 004; móvil: +34-

619222 209; fax: +34-987 291

004

buff@ unileon.es

\section{Desde un archivo}

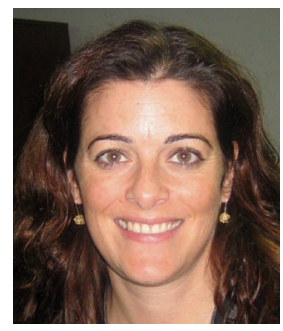

Tania Cid Gonzólez, Archivo General de Castilla y León Valladolid.

El ámbito de los archivos, como unidades o centros de información que son, es uno más al que los profesionales de nuestro campo pueden acceder $\mathrm{y}$, sin embargo, resulta ser generalmente el menos explorado por parte de los diplomados y licenciados que emergen al mundo laboral tras su paso por la Universidad.

Y ello a pesar de que la formación en materia archivística que nuestra titulación (tanto la Diplomatura como la Licenciatura) nos facilita tiene un peso similar con respecto a la de las otras dos ramas de nuestra disciplina, la Biblioteconomía y la Documentación, como se constata a la hora de contabilizar el número de créditos que corresponderían a asignaturas relacionadas con los archivos: por ejemplo, de un total de 206 créditos de la Diplomatura $^{1}$ el alumno interesado en especializarse en Archivística pue-

\section{"En principio, los diplomados y licenciados en ByD están suficientemente preparados para ejercer en cualquier tipo de archivo"}

de cursar 68 créditos; y en el caso de la Licenciatura ${ }^{2}$, de los 120 créditos totales 25 están directamente relacionados con los archivos, a los que se sumarían los 20 créditos que cada alumno puede elegir libremente. Así pues, se puede decir a priori que un diplomado o un licenciado estaría suficientemente preparado para iniciar su vida laboral en cualquier tipo de archivo y que la formación académica, en este sentido, parece adecuada.

Ahora bien, el acceso -mediante oposición- a puestos de trabajo en archivos del sector público supone, para estos titulados, un doble e intenso esfuerzo dada su especificidad: los temarios de los procesos selectivos (tanto los de Facultativo como los de Ayudantes de Archivos) incluyen un número elevado de temas de carácter histórico (en algunos casos superando los de archivística), a la vez que exigen un conocimiento exhaustivo de técnicas paleográficas -imprescindibles para superar el ejercicio práctico- y el manejo de, al menos, un idioma (habitualmente inglés o francés, aunque en las oposiciones en las que se requieren dos idiomas también es posible elegir una lengua clásica-latín o griego).

\section{"El esfuerzo que requiere presentarse a una oposición de archivos bien merece la pena, dada la mínima competencia en número de aspirantes"}

Por lo tanto, el titulado que aspira a ingresar en alguno de los cuerpos de Archiveros o Ayudantes de archivos de la Administración, tanto autonómica como estatal, no solamente deberá contar con un buen temario (normalmente de elaboración propia) sino que se verá obligado a adquirir ciertas habili- dades y destrezas que le permitan superar con éxito una oposición de este tipo. Y es precisamente aquí, en la práctica de las técnicas historiográficas, donde el diplomado en biblioteconomía y documentación o el licenciado en documentación se muestra en inferioridad de condiciones con respecto, por ejemplo, a los licenciados en Historia que tradicionalmente se han venido presentando a estas oposiciones.

Como conclusión cabría destacar que, si bien la formación en materia archivística de nuestros planes de estudios se presenta coherente y ajustada a la realidad del trabajo desempeñado en un archivo, lo cierto es que acceder a un puesto de funcionario en cualquiera de los archivos de la Administración pública se vuelve, para los diplomados y licenciados, una cuestión de voluntad, esfuerzo y preparación extra universitaria que, por otra parte, bien merece la pena dada la mínima competencia (en cuanto al número de aspirantes) con que uno se encuentra a la hora de examinarse.

\section{Notas}

1. Se ha tomado como referencia el vigente plan de estudios de la Diplomatura en biblioteconomía y documentación de la Universidad de León.

2. El plan de estudios que se ha contabilizado corresponde a la Licenciatura en documentación ( $2^{\circ}$ ciclo) de la Universidad de Salamanca.

\section{Tania Cid González}

Archivo General de Castilla y León Palacio del Ldo. Butrón, Pza. Sta.

Brígida, $s / n$

47003 VALLADOLID

Tel.: 983317 746; fax: 983412 919

cid_tania@yahoo.es, cidgonta@ jcyl.es

\section{Desde la empresa privada}

Como diplomada en biblioteconomía y documentación por la Universidad de León y licenciada en documentación por la Universidad Carlos III de Madrid, tiene una 


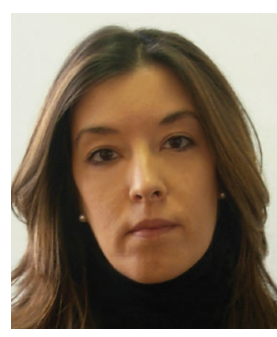

AlejandrafolgadoMajo, Departamento de Documentación de Irea (Madrid)

buena perspectiva sobre la formación y también sobre el ejercicio de la profesión, pues acumula casi 10 años de experiencia en centros de documentación de empresa.

El hecho de haber trabajado siempre en el ámbito de la empresa privada, y en concreto en el mercado del asesoramiento financiero y estratégico, le ha proporcionado una importante visión analítica que le ayudó bastante a la hora de tener que elaborar una critica tanto de la formación como el papel que tiene el profesional de la información resultante de esa formación.

Este es el análisis Dafo (debilidades, amenazas, fortalezas y oportunidades) que presentó:

- Fortalezas: Formación multidisciplinar, balance adecuado entre teoría y práctica. Desarrollo de la capacidad de relación en el profesional, visión de $360^{\circ}$ para la gestión de documentación sea cual sea su soporte.

- Debilidades: En ocasiones una formación "dispersa" que aporta poco valor al desarrollo profesional. Descompensación formativa, el estudiante recibe mucha formación en unos campos y poca en otros. Distancia entre la formación recibida y la profesión que tiene que ejercer.

- Amenazas: Que otras titulaciones comiencen a suplirnos en nuestras funciones. Que la falta de conocimiento de nuestras posibilidades merme nuestro desarrollo. No ser competitivos por tener una formación poco adecuada al momento social. No ser capaces de demostrar nuestro valor como profesionales por desconocimiento de las acciones y herramientas necesarias para ello.

- Oportunidades: Conocer de primera mano la opinión de quien se ha formado profesionalmente a partir de aquellos planes de estudio y poder corregir deficiencias. Diálogo permanente entre profesionales y docentes. El profesional tiene la oportunidad de desarrollar su carrera en 3 campos (bibliotecas, archivos y unidades de información) y en cualquier área de especialización. Puede contribuir a consolidar la profesión mediante la aportación de valor en el centro donde realice su trabajo.

El análisis Dafo resultante pone de manifiesto que dichas fortalezas y debilidades, se inician en la formación y convergen en la profesión. Revela amenazas pero también identifica oportunidades clarísimas tanto para los docentes como para los profesionales.

Por tanto, se requiere una formación en biblioteconomía y documentación que sea capaz de mantener el ritmo de evolución de la sociedad de la información para obtener profesionales competitivos que asuman la responsabilidad de aportar opiniones y soluciones a los problemas formativos, ya que tienen tanto la visión crítica como las herramientas de mejora. "Es un ejercicio de responsabilidad en aras de la consolidación de nuestra profesión en España”- terminó Folgado.

\section{Alejandra Folgado, directora de Organización \\ Irea, $P^{o}$ de la Castellana, 141. Ma- drid 28046 \\ Tel.: +34-915 798 400; fax: +34- 915723207 \\ http://www.irea.es \\ alejandra.folgado@irea.es}

\section{Desde la administración pública autonómica}

"Los profesionales de la información debemos ir dejando a un

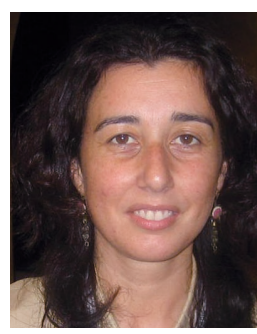

Raquel LavanderaFernández, Oficina de Investigación Biosanitaria (Ficyt), del Principado de Asturias.

lado nuestros complejos y creernos, de una vez por todas, nuestra valía. La palabra 'no' nunca debe figurar en nuestro vocabulario, estamos capacitados y preparados para afrontar todos los retos que nos propongamos" -fue la optimista introducción de Lavandera.

Continuó señalando que nuestro perfil es amplio, por lo que en general tenemos que tender a la especialización y nunca dejar de lado la autoformación. Debemos ir con los tiempos, con las TIC, con la web 2.0, con nuestros usuarios, siempre por delante y pensando en positivo. Trabajar en equipo y unir esfuerzos también es necesario, en ocasiones la colaboración con los técnicos en tecnologías de la información resulta imprescindible.

Los planes de estudio, nuestras tareas diarias, incluso nuestras herramientas de trabajo, han cambiado, y siguen evolucionando, pero no nos asustamos por ello, todo lo contrario, pues nos hemos acostumbrado a afrontar nuevos retos.

\section{"Tal vez deberíamos dejarnos de tanto análisis de la profesión y empezar a creer en nosotros mismos"}

"También es verdad que al poder hacer tantas cosas en ocasiones puede parecer que ni nosotros mismos seamos capaces de saber para qué servimos: lo mismo gestionamos webs o bases de datos, 
que catalogamos libros o ejercemos de psicólogos de nuestros usuarios, aunque también podemos diseñar actividades de animación a la lectura, de e-learning o de alfabetización informacional, preparar informes sobre las materias más diversas, diseñar estrategias de búsqueda, recopilar bibliografías, indizar o preparar resúmenes, analizar motores de búsqueda, gestionar catálogos, blogs, listas de distribución...

Aunque también podemos morirmos de asco explicando al jefe, político o gestor de turno, por enésima vez, qué es un 'profesional de la información'. En las Jornadas Fesabid de 1994, en Gijón, se repartieron una camisetas en las que ponía: 'Soy documentalista' y por la parte de atrás se podía leer ‘Docu qué?'; 14 años después esa camiseta sigue estando de actualidad (yo aún la tengo).

Lo que sí tengo claro es que no me arrepiento de haber elegido esta profesión, hoy en día volvería a estudiar biblioteconomía y documentación, sin ninguna duda".

Raquel Lavandera Fernández, documentalista, Oficina de Investigación Biosanitaria (Ficyt).

González del Valle, 2 - $1^{o}$

33003 Oviedo

Tel.: +34-985964595

raquellf@ficyt.es, raquellf@princast.es

\section{Desde una fundación}

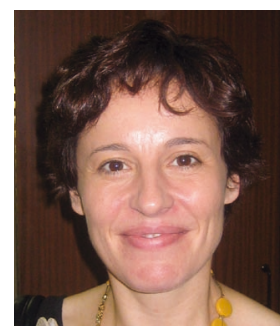

Esther Sánchez Coro Centro de Documentación "Antonio del ValleMenéndez", Fundación Hullera Vasco-Leonesa, La Robla, León.

En estos últimos años todos los aspectos relacionados con la gestión de la información han evolucionado de una manera vertiginosa. Los cambios tecnológicos, a los que se añaden los sociales y económicos, han convertido en caduca la Universidad que conocimos hace 15 años.

Amparo Moraleda, presidenta de IBM para el Sur de Europa, declaraba recientemente en un periódico de tirada nacional que "en tres años queda obsoleta la mitad de la formación que se recibe en las universidades".

En su opinión, hay dos aspectos que actualmente debe tener en cuenta la universidad como entidad generadora de talento:

1. Flexibilidad para adaptarse a un mundo en constante movimiento. Hay estudios que reflejan datos tan contundentes como que los empleos especializados que serán más demandados en 2010 simplemente no habrían existido seis años antes debido, sobre todo, al rápido avance tecnológico.

2. Potenciación de la colaboración Universidad-Empresa debido a que la innovación se crea, fundamentalmente, a partir de este binomio. Hay mucho que hacer para construir una relación que, en demasiadas ocasiones, no ha funcionado por desconfianza de ambas partes.

Otra de las asignaturas pendientes de la Universidad es formar a sus alumnos en competencias que demanda el mercado laboral, entre las que se encuentran:

1. Tecnología. En el caso de los profesionales de la información es imprescindible un adecuado conocimiento de los sistemas de gestión documental más comunes, de lenguajes de programación, diseño y gestión de bases de datos y diseño de aplicaciones web.

2. Idiomas. Es fundamental un buen nivel de inglés y, si a éste se suma el conocimiento de otro/s idioma/s, el valor añadido con el que va a contar el profesional es innegable.

\section{Competencias personales} y relacionales que se van a encontrar en el puesto de trabajo, como capacidad de negociación; conocimientos de oratoria tan necesarios a la hora de realizar presentaciones en público; enseñar qué es la empatía; trabajo en equipo; espíritu emprendedor; formación en materia de liderazgo (perder el miedo a tomar decisiones, liderar proyectos...); Cuando estas competencias se trasladen al alumnado surgirán profesionales que innovan, que motivan, que tienen ideas, en definitiva, que saben hacia donde quieren ir.

Además, se debería abolir la actitud pasiva del alumno en las clases. Si se necesita dar / recibir apuntes sería de gran utilidad habilitar un espacio web que haga posible su descarga con objeto de que el alumno se los estudie en orden a poder aprovechar adecuadamente unas clases eminentemente prácticas, ya que el mercado requiere una formación útil, con ideas y soluciones a problemas reales.

También sería muy positivo el debate de ideas desde el primer curso, ya que discutir agiliza la mente $\mathrm{y}$, a su vez, genera ideas nuevas y soluciones. Se debería valorar más que los alumnos se arriesguen con hipótesis nuevas, cambiar la concepción de error que se tiene en España y seguir la tendencia de países como Estados Unidos donde se valora el error porque eso significa innovación, más experiencia. Si cambiásemos esta concepción tanto alumnos como profesores se sentirían con más libertad de actuación.

Todo ello, por supuesto, debe estar acompañado de una capacidad autónoma de esfuerzo personal y de aprendizaje por parte del alumno, que tan importante va a ser en su posterior vida laboral y en la necesaria formación continua para un adecuado desempeño profesional.

Asimismo es fundamental un correcto uso del lenguaje, tanto oral 
como escrito, por parte de cualquier profesional, y más aún si cabe, en el caso de los profesionales de la información. No debemos olvidar que proporcionar información con faltas de ortografía, mal expresada o mal presentada no sólo dificulta el desempeño de nuestra profesión, al no ser capaz de transmitir convenientemente el mensaje que deseamos, sino que devalúa seriamente al profesional que lo ha realizado.

Si los profesionales de la información logramos una formación sólida en los aspectos relacionados anteriormente, su futuro estará tanto en archivos, bibliotecas, centros de documentación tradicionales, como en los medios de comunicación y en el mundo de la empresa en general, tanto externa como autónoma, a lo que habría que sumarle las posibilidades del teletrabajo.

Un mundo lleno oportunidades para una profesión con mucho futuro, ya que para cualquier organización que quiera ocupar una posición de liderazgo en un mundo altamente competitivo y en constante cambio es vital saber hallar la información idónea.

Esther Sánchez Coro, Centro de Documentación "Antonio del Valle Menéndez”, Fundación Hullera Vasco-Leonesa.

Avda. La Térmica, 2. 24640 La Robla, León.

Tel.: /fax: +34-987570160

centrodoc@fhvl.es

\section{Desde la universidad}

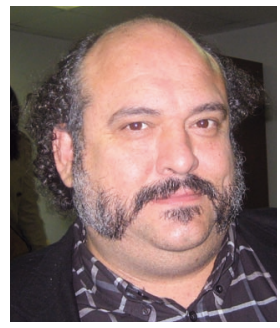

Felipe Zapico Alonso, profesor de Biblioteconomía y Documentación de la Universidad de Extremadura.

"Con respecto a las fortalezas y debilidades, y resumiendo, diré que son las mismas, nuestras fortalezas son nuestras debilidades y viceversa".

Me explicaré con mi caso, la formación que he recibido. El plan de estudios que cursé durante la Diplomatura en Biblioteconomía y Documentación en la primera promoción en la Universidad de León (1990-1993) era nefasto: por poner un ejemplo diré que había una asignatura denominada Documentación I que en realidad era Diplomática General, por lo que la Documentación nos la tuvimos que buscar los alumnos. El plan de estudios de la Licenciatura en Documentación que cursé en la Universidad de Salamanca, también en la primera promoción (1994-1996), tampoco fue para tirar cohetes.

De cualquier forma, la formación inicial no es determinante, además de que los planes de estudio han ido cambiando, adaptándose y cambiando el profesorado. Pero el hecho de que en muchas facultades y ámbitos siempre se nos haya relacionado con las Humanidades, ha resultado que se nos ha asimilado a esa formación sin más, sin contemplar las muchas ampliaciones de que gozamos.

Podemos realizar un sinfín de funciones, y creo que la formación acaba siendo todo aquello que vamos incorporando a nuestro conocimiento cada día: todas las asignaturas (buenas y malas), cursos, lecturas, la navegación diaria, nuestro trabajo. Todo eso de que somos capaces es la mayor fortaleza, ya que la premisa de los profesionales de la información debe ser una curiosidad insaciable.

Yo continué la formación con un curso de Posgrado en Sistemas de Información y la concesión del grado de Doctor en la Universidad de Salamanca en 2001. Todo ello con numerosos cursos de formación y perfeccionamiento.

Ocupé un gran número y variedad de puestos de trabajo en As- torga, León y Salamanca (incluso yendo y viniendo diariamente entre estas dos ciudades), hasta que finalmente me asenté felizmente en la Universidad de Extremadura, en Badajoz, donde trabajo a día hoy como profesor titular. También pertenezco al Grupo Scimago de investigación.

Felipe Zapico Alonso, Facultad de Biblioteconomía y Documentación, Universidad de Extremadura.

Plazuela Ibn Marwam, s/n. 06071 Badajoz.

Tel.: +34-924 289300 (6422); fax: +34-924 286401

fzapalo@alcazaba.unex.es

\section{Desde la administración pública central}

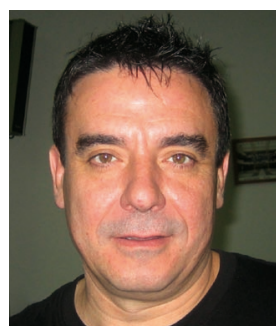

Juan Carlos Pajares Iglesias, Archivo de la Subdelegación del Gobierno (León) y profesor asociado de Biblioteconomía y Documentación de la Universidad de León.

La formación de los profesionales de la información en las universidades es adecuada y suficiente, pero hay que tener en cuenta que la adecuación de los planes de estudio a los diferentes y habituales cambios a los que se ve sometido el entorno profesional no siempre es inmediata. Los cambios de los planes de estudio requieren de unos procesos burocráticos que dilatan su implantación y a veces son cautivos de causas que poco o nada tienen que ver con cuestiones puramente académicas.

Aun así, mi experiencia como titulado de la primera promoción de la diplomatura en biblioteconomía y documentación de la Universidad de León y en la primera promoción de la licenciatura en documentación por la Universidad de Salamanca (curso 1994-1996), me demuestra que a pe- 
sar de ser ambos planes de estudios mejorables y mejorados, me aportaron los conocimientos y las técnicas adecuadas para desempeñar mi labor como profesional de la información en el mercado laboral.

La formación universitaria no puede ser nunca la única que asegure la total aptitud de un profesional para su vida laboral. En cualquier área del conocimiento y quizás, al menos en las últimas décadas, más en la nuestra, la formación continua es ineludible y absolutamente necesaria. Formación que programen las empresas e instituciones, y formación procurada por los propios profesionales, en función de sus carencias e intereses.

La labor personal en este aspecto es fundamental, de la misma manera que lo es la búsqueda de oportunidades de trabajo. No siempre las instituciones públicas o las empresas (es el caso de la comunidad autónoma de Castilla y León, a pesar de contar con dos sedes universitarias donde se imparten los estudios de la diplomatura y de la licenciatura) demuestran una sensibilidad adecuada con nuestra profesión. Es importante que el titulado indague en las oportunidades de trabajo que no están explicitadas, ofreciendo sus capacidades, anticipándose a las necesidades de las organizaciones.

"El titulado debe indagar
las oportunidades de
trabajo que no están
explicitadas, ofreciendo
sus capacidades y
anticipándose a las
necesidades de las
organizaciones"

\section{Conclusiones de la segunda mesa redonda:}

- La principal fortaleza residía en la formación técnica recibida tanto del ámbito de los archivos como de las bibliotecas.

- La debilidad a subsanar en el próximo plan de estudios de Grado es la formación tecnológica, que deber ser más profunda, así como dotar al alumnado de conocimientos del entorno empresarial.

- Los ponentes aconsejaron también a los estudiantes y egresados más recientes que asumiesen funciones en las organizaciones y se mostrasen siempre dispuestos a desempeñar las variadas tareas que a un profesional de la información se le pueden encargar.

"Las universidades deben formar profesionales que se desenvuelvan perfectamente en los tres ámbitos: bibliotecas, archivos y centros de documentación, o lo que es lo mismo, que manejen con destreza las técnicas y las herramientas bibliotecarias, archivísticas y documentales. La empresa privada, que es el mercado natural y por explotar al que deberían abocarse los titulados, precisa cada vez más lo que vengo en denominar los "tres en uno" (un profesional polivalente) y por eso veo muy adecuado el cambio de denominación de los estudios como Grado en documentación e información. El término biblioteconomía capitalizaba en exceso la visión que la sociedad en general tiene de estos títulos, y por otra parte recuperamos el término información que nos había sido sustraído. A veces las denominaciones y las etiquetas tienen más influencia en la sociedad de la que suponemos.

Juan Carlos Pajares Iglesias, Archivo de la Subdelegación del Gobierno (León); profesor asociado de Biblioteconomía y Documentación de la Universidad de León. Plaza de la Inmaculada, 6. 24071 León.

Tel.: +34-987 969 118; fax: 987 969154

juancarlos.pajares@map.es

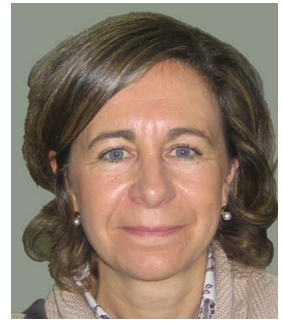

Moderadora de la $2^{\mathrm{a}}$ mesa redonda, Lourdes Santos de Paz es profesora titular de $\mathrm{Bi}$ blioteconomía y Documentación de la Universidad de León. Su perfil docente e investigador es la biblioteconomía y la conservación de documentos.

Los ponentes fueron críticos con algunas de las materias que habían cursado en su formación tanto en León como en las universidades en que habían realizado la licenciatura. Eran conscientes, no obstante, de que había transcurrido más de una decena de años desde que terminaron sus titulaciones y que la formación actual es más acorde con las necesidades del mercado laboral.

Más información:

http://www3.unileon.es/dp/abd/

Blanca Rodríguez-Bravo, Universidad de León, Facultad de Filosofía y Letras, Campus Vegazana, 24071 León.

Tel.: +34-987 291 469; fax: +34987291863

dphbrbunileon.es 